\title{
Physics, complexity and causality
}

\author{
Although the laws of physics explain much of the world around us, we still do not have a realistic \\ description of causality in truly complex hierarchical structures.
}

\section{George F. R. Ellis}

The atomic theory of matter and the periodic table of elements allow us to understand the physical nature of material objects, including living beings. Quantum theory illuminates the physical basis of the periodic table and the nature of chemical bonding. Molecular biology shows how complex molecules underlie the development and functioning of living organisms. And neurophysics reveals the functioning of the brain.

In the hierarchy of complexity, each level links to the one above: chemistry links to biochemistry, to cell biology, physiology, psychology, to sociology, economics, and politics. Particle physics is the foundational subject underlying - and so in some sense explaining - all the others. In a reductionist world view, physics is all there is. The cartesian picture of man as a machine seems to be vindicated.

But this view omits important aspects of the world that physics has yet to come to terms with. Our environment is dominated by objects that embody the outcomes of intentional design (buildings, books, computers, teaspoons). Today's physics has nothing to say about the intentionality that has resulted in the existence of such objects, even though this intentionality is clearly causally effective.

A simple statement of fact: there is no physics theory that explains the nature of, or even the existence of, football matches, teapots, or jumbo-jet aircraft. The human mind is physically based, but there is no hope whatever of predicting the behaviour it controls from the underlying physical laws. Even if we had a satisfactory fundamental physics 'theory of everything,' this situation would remain unchanged: physics would still fail to explain the outcomes of human purpose, and so would provide an incomplete description of the real world around us.

Can we nevertheless claim that the underlying physics uniquely causally determines what happens, even if we cannot predict the outcome? To examine whether we can, contemplate what is required for this daim to be true within its proper cosmic context. The implication is that the particles existing when the cosmic background radiation was decoupling from matter, in the early Universe, were placed precisely so as to make it inevitable that 14 billion years later, human beings would exist, Charles Townes would conceive of the laser, and Edward Witten would develop string theory. Is it plausible that quantum fluctuations in the inflationary era in the very
With this view, the higher levels in the hierarchy of complexity have autonomous causal powers that are functionally independent of lower-level processes. Topdown causation takes place as well as bottom-up action, with higher-level contexts determining the outcome of lowerlevel functioning, and even modifying the nature of lower-level constituents.

Stored information plays a key role, resulting in non-linear dynamics that are non-local in space and time. Brain functioning is causally affected by abstractions, such as the value of money, the rules of chess and the theory of the laser. These abstractions are realized as brain states in individuals, but are not equivalent to them - James Clerk Maxwell's theory of electromagnetism is not the same as any individual's brain state. Although such concepts are causally effective, they are not themselves physical variables. Consequently physics per se cannot causally early Universe - the source of the perturbations at the time of decoupling implied the future inevitability of the Mona Lisa and Einstein's theory of relativity? Those fluctuations are supposed to have been random, which by definition means without purpose or meaning.

However, such meaning did indeed come into being. Ever higher levels of interaction and causality arose as complexity spontaneously increased in the expanding Universe, allowing life to emerge. Darwinian processes of selection guided the physical development of living systems, including the human brain.

It is possible that what actually happened was the contextual emergence of complexity: the existence of human beings and their creations was not uniquely implied by the initial data in the early Universe; rather the underlying physics together with that initial data created a context that made the existence of human beings possible. Conditions at the time of the decoupling of matter and radiation 14 billion years ago were such as to lead to the eventual development of minds that are autonomously effective. Such minds are able to create higher-level order, such as the Hubble Space Telescope and Kurt Godel's incompleteness theorem, that embodies a purpose and meaning not in existence before. determine the outcome of human creativity; rather it creates the 'possibility space' to allow human intelligence to function autonomously.

This situation is not dependent specifically on human intentionality. Physics by itself cannot explain anybehaviour that is adaptive and depends on context, for example, beaver dam-building and the dances of bees. It is plausible that these also emerged at late times in the expanding Universe as higher-level autonomous behaviours, made possible but not causally determined by the underlying physics and chemistry of matter.

If this is the case, the challenge to physics is to develop a realistic description of causality in truly complex hierarchical structures, where top-down causation and memory effects allow autonomous higher levels of order to emerge with genuine causal powers. So far, attempts to relate physics to complexity - such as the reaction-diffusion equation, chaos theory, the renormalization group, complexity theory - take us only a small step on this road.

George F.R. Ellis is at the Mathematics

Department, University of Cape Town, Rondebosch, Cape Town 7701, South Africa.

FURTHER READING

Ellis, G.F. R. Fhys Today (in the press).

Bishop R. C. Phil. Sci. (in the press). 